\title{
Are antibiotics being used appropriately for emergency dental treatment?
}

\author{
Y. M. Dailey, ' and M. V. Martin, ${ }^{2}$
}

\begin{abstract}
Aim To investigate the therapeutic prescribing of antibiotics to patients presenting for emergency dental treatment.

Design A prospective clinical study.

Method Information was collected via a questionnaire concerning the patient's reason for attendance and treatment undertaken at emergency dental clinics in North and South Cheshire.

Results Over an 11-week period 1,069 patients attended the five clinics, 1,011 questionnaires were analyzed. The majority of the attendees had pain (879/1011).35\% (311/879) of these patient had pulpitis and $74 \%(230 / 311)$ had been issued a prescription for antibiotics, without any active surgical intervention. The principal antibiotic prescribed for both adult and child patients was amoxicillin.

Conclusion The majority of patients attending the emergency dental clinics had pain, with a large proportion having localised infections either as pulpitis or localised dental abscess. Three quarters of these patients had no surgical intervention and were inappropriately prescribed antibiotics.
\end{abstract}

$\mathrm{T}$ he General Dental Council states that 'the dentist has a professional responsibility for emergency dental care'. They recognise the difficulties in defining an emergency but state that a sympathetic response to patients with pain is expected. If a patient has acute spread of infection, haemorrhage, or trauma, it is the dentist's duty to make arrangements for the patient to receive advice or treatment in a reasonable time. ${ }^{1}$

Management of acute dental conditions is primarily based upon extraction of teeth or extirpation of the pulp. ${ }^{2}$ The use of antibiotics as an adjunct in the management of orofacial infections is an important treatment option and when clinically indicated is of therapeutic benefit to the patient. ${ }^{3}$ However systemic antibiotics should be used with restraint because of the possibility of allergic reactions, toxicity, side effects and the development of resistant strains of microbes. ${ }^{4}$

In 1996 Thomas et al., investigated the prescribing of antibiotics to emergency dental patients by primary healthcare workers. ${ }^{5}$ They concluded that both general medical and general dental practitioners had prescribed antibiotics inappropriately to patients with dental emergencies. Evidence from further studies further suggests that antibiotics are being prescribed inappropriately within general dental practice. ${ }^{6-9}$ However these studies are based upon hypothetical case scenario questionnaires. They are unable to predict the effect

$1^{*}$ Lecturer, ${ }^{2}$ Senior Lecturer, Department of Clinical Dental Sciences, University of Liverpool, Liverpool L69 3BX

${ }^{*}$ Correspondence to: $Y$. M. Dailey

email: Y.M.Dailey@liverpool.ac.uk

REFEREED PAPER

Received 21.02.00; Accepted 15.05.01

(C) British Dental Journal 2001; 191:391-393 that the dentist-patient relationship may have upon the practitioners' prescribing behaviour. The aims of this study were to investigate the appropriateness of antibiotic prescribing to patients presenting for emergency dental treatment.

\section{Materials and methods}

The study was undertaken prospectively over an 11-week period, between January 1999 and April 1999, at five out-of-hours emergency dental clinics provided by North and South Cheshire Health Authorities. The timing of the study was set to avoid any bank holidays, when it was known that the number of patients attending the clinics increased. The dentists providing the emergency treatment collected information for each patient who attended the clinic.

\section{Data Collection}

Information about the patient's reason for attending the emergency clinic and the treatment provided was collected using a questionnaire. The dental nurse completed the first part of the questionnaire; which recorded patient's demographic details including gender, date of birth and whether the patient claimed to be registered with a dentist. The dentist completed the rest of the questionnaire, which recorded the nature of the patient's complaint. The complaints were classified as pain, localized swelling, diffuse swelling, swelling that caused closure of the eye or difficulty in swallowing, lost restorations, cavities, trauma, and bleeding, A space was left for the dentist to record their clinical diagnosis of the patient's complaint and the treatment provided. If the treatment involved the prescribing of antibiotics the dentist was asked to indicate the type of antibiotic prescribed.

\section{Statistical analysis}

The questionnaires received were coded and entered into the Statistical Package for Social Sciences (SPSS) Version 9 for windows TM. ${ }^{10}$ Summary statistics were calculated to include frequencies, percentages, and means and standard deviations where indicated.

\section{Results}

During the study period 55 dentists worked in the emergency dental clinics, from 10.00 am until 12.00 midday, on both Saturdays and Sundays. There were 34 male and 21 female dentists. Their mean age was 41 years (range 27-56). A total of 1,069 patients attended the five emergency dental clinics over the 11-week period. Fifty-eight questionnaires were incomplete and the remaining 1,011 were analysed.

The mean number of patients attending the clinics per session was 10.2 (range 6-31). There were 522 males $(52 \%)$ and 489 females (48\%), of which $895(89 \%)$ were adults and $116(11 \%)$ children (i.e. below the age of 18 years). A total of 91 ( $9 \%)$ patients attending the clinics were thought by the dentists to be non-genuine emergencies: those with lost crowns/bridges and one patient requesting a new pair of dentures. 


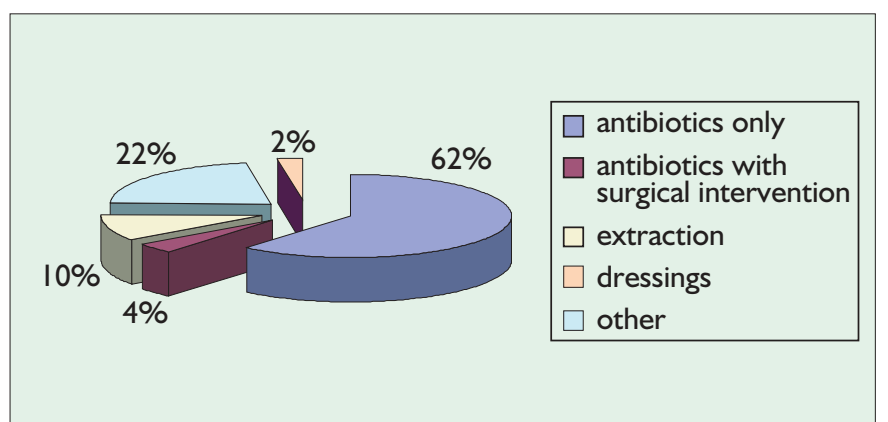

Fig. I Treatment received by adult patients attending the emergency dental clinics

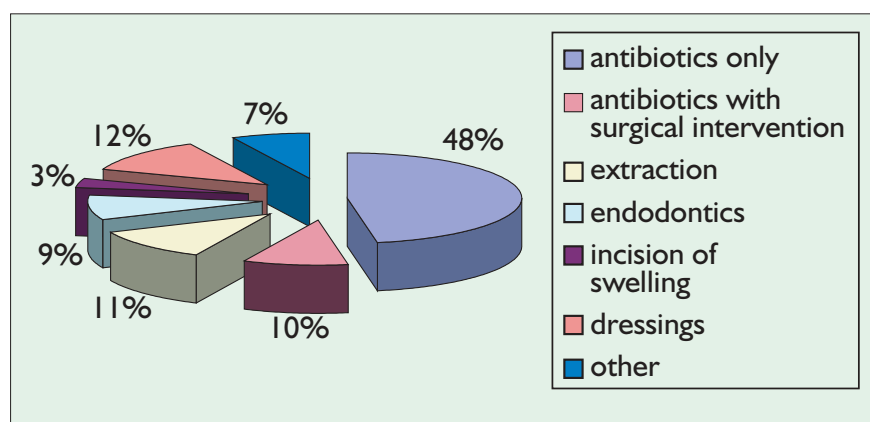

Fig. 2 Treatment received by child patients attending the emergency dental clinics
Reasons for attendance

Table 1 shows the reasons for attendance at the emergency dental clinics. The majority of patients 887 (adult: 788/895, 88\%, child: $99 / 116,85 \%)$ presenting at the emergency clinics complained of pain. In some cases other signs and symptoms were also present. One hundred and five (adult: 82/895, 9\%, child: 23/116, 20\%) had a localized swelling, whilst 51 (adult: 49/895, 5\%, child: 2/116, 1.5\%,) had a diffuse swelling, 3 adults had swelling causing difficulty in swallowing and 2 had swelling which closed the eye. The proportion of patients who were recorded as having a cavity (adult: $72 / 895,8 \%$, child: $26 \%, 31 / 116$ ) or dental trauma (adult: $2 / 895,0.2 \%$, child: $5 / 895,4 \%)$ was higher in children than adults. However one adult had presented with facial trauma and another with a bleeding socket, following a tooth extraction 36 hours earlier.

\section{Treatment Provided}

Figures 1 and 2 show the treatment received by adult and child patients attending the emergency dental clinics. The issuing of a prescription was the only treatment that 495 (49\%) of adult and 77 $(62 \%)$ of child patients received, at the emergency dental clinics. A higher proportion of adults $(86 / 895,10 \%)$ than children $(5 / 116$, $4 \%)$ received active surgical treatment together with the antibiotic prescription.

The proportion of patients undergoing extraction without the issue of a prescription was similar for both adults and children (adults: $101 / 895,11 \%$, child: 13/116 10\%). Only adults however, had their swelling incised or underwent endodontic treatment. Dressings were provided for lost restorations, tooth fractures and dry sockets. Sixty-five adults received a variety of treatments, which included recementing of crowns/bridges; curettage around

Table I Clinical signs and symptoms associated with patients' attendance at the emergency dental clinics

\begin{tabular}{|c|c|c|}
\hline Signs and symptoms & $\begin{array}{l}\text { Adult patients } \\
n=895\end{array}$ & $\begin{array}{c}\text { Child patients } \\
n=116\end{array}$ \\
\hline Pain & $788(88 \%)$ & $99(85 \%)$ \\
\hline Localised swelling & $82(9 \%)$ & $23(50 \%)$ \\
\hline Cavities/fracture & 72 (8\%) & $31(26 \%)$ \\
\hline Lost restorations & $64(7 \%)$ & $5(4 \%)$ \\
\hline Diffuse swelling & $49(5 \%)$ & $2(1.5 \%)$ \\
\hline Lost crowns/bridges & $45 \quad(5 \%)$ & 0 \\
\hline Gingival bleeding & $26(3 \%)$ & I (0.8\%) \\
\hline Swelling and eye closure & $2(0.2 \%)$ & 0 \\
\hline Swelling and difficulty in swallowing & $3(0.3 \%)$ & 0 \\
\hline \multicolumn{3}{|l|}{ Trauma } \\
\hline Facial & I (0.1\%) & 0 \\
\hline Dental & $2(0.2 \%)$ & $5(4 \%)$ \\
\hline Persistent hemorrhage & I $(0.1 \%)$ & 0 \\
\hline Other* & $36(4 \%)$ & $3(2 \%)$ \\
\hline
\end{tabular}

*Respondents were able to indicate more than one sign or symptom partially erupted eight's and advice only. Three of these patients were referred to the local hospital oral surgery department. This included a patient with a suspected condyle fracture, and two patients who had difficulty in swallowing because their swelling was crossing the midline.

Prescribing of antibiotics for pain (Table 2)

Dentoalveolar abscess and pulpitis was attributed to the cause of pain in nearly all child patients $(94 / 99,95 \%)$, three quarters of these children received an antibiotic prescription $(76 / 99,76 \%)$. The same diagnosis was attributed to over half of the adult patients with pain $(464 / 788,59 \%)$, of whom nearly half received an antibiotic prescription $(355 / 788,45 \%)$.

Sixty-nine $(7.8 \%)$ adult patients, who complained of pain had a periapical infection related to ongoing or completed root canal treatment, 16 of them received a prescription for antibiotics. A

Table 2 Clinical diagnosis of patients' pain for which antibiotics were prescribed

\begin{tabular}{lcccc}
\hline & \multicolumn{2}{c}{ Adult patients } & \multicolumn{2}{c}{ Child patients } \\
Diagnosis & $\begin{array}{c}\text { Number } \\
\text { with pain }\end{array}$ & $\begin{array}{c}\text { Number } \\
\text { prescribed } \\
\text { antibiotics }\end{array}$ & $\begin{array}{c}\text { Number } \\
\text { with pain }\end{array}$ & $\begin{array}{c}\text { Number } \\
\text { prescribed } \\
\text { antibiotics }\end{array}$ \\
\hline Pulpitis & 268 & 195 & 43 & 35 \\
Dentoalveolar abscess & 196 & 160 & 52 & 41 \\
Periapical infection & 56 & 7 & - & - \\
$\quad$ During root treatment & 56 & 9 & - & - \\
After root treatment & 13 & 21 & - & - \\
Pericoronitis & 41 & 31 & - & - \\
Periodontal abscess & 41 & 25 & - & - \\
Acute ulcerative gingivitis & 39 & 14 & - & - \\
Dry socket & 36 & 16 & - & - \\
Cellulitis & 16 & 2 & - & - \\
Sinusitis & 6 & 1 & 5 & I \\
Trauma & 3 & 28 & - & - \\
Unable to diagnose & 64 & 509 & 99 & 77 \\
\hline Total & 788 & & & \\
\hline
\end{tabular}

Table 3 Type of antibiotic by GMPs and dentists

\begin{tabular}{lcc}
\hline Antibiotic & $\begin{array}{c}\text { Number of patients } \\
\text { receiving antibiotic }(n=586)\end{array}$ & $\begin{array}{c}\text { Percentage of } \\
\text { prescriptions issued }\end{array}$
\end{tabular}

\begin{tabular}{lrr} 
Amoxicillin & 422 & $(72.0 \%)$ \\
Amoxicillin and Metronidazole & 78 & $(13.3 \%)$ \\
Metronidazole & 45 & $(7.7 \%)$ \\
Penicillin V & 29 & $(4.9 \%)$ \\
Erythromycin & 11 & $(1.9 \%)$ \\
Erythromycin and Metronidazole & $\mathrm{I}$ & $(0.2 \%)$ \\
\hline Total & 586 & $100 \%$ \\
\hline
\end{tabular}


definitive diagnosis could not be made for 64 (7.3\%) of the patients who attended the clinic complaining of pain, just over one third $(24 / 64)$ received a prescription for antibiotics.

\section{Antibiotics prescribed}

Table 3 shows the frequency of the antibiotics prescribed. For both adults and children amoxicillin was the most frequently prescribed antibiotic $(422 / 509,72 \%)$. Only adult patients were prescribed a combination of metronidazole and amoxicillin: 78 (13.3\%). A total of $45(7.7 \%)$, adults were prescribed metronidazole only . The prescribing of penicillin and erythromycin was infrequent; only one adult patient received a combination of erythromycin and metronidazole.

\section{Discussion}

Ninety five per cent of the questionnaires returned were completed. To encourage cooperation with data collection, the questionnaire was designed to be brief and easy to complete.

The study investigated the prescribing of antibiotics during emergency dental treatment in North and South Cheshire. The authors acknowledge that the results may not be a representative sample of the UK. The levels of prescribing could be affected by differences in service provision and the type of dental emergency presenting. Nevertheless the results confirm the main findings of recent questionnaire studies, in general dental practice the fundamental principles in antibiotic prescribing are being ignored and antibiotics are being inappropriately prescribed

The majority of the patients attending the emergency clinics were complaining of pain. For nearly all the child patients and three quarters of the adult patients, pain was attributed to a localised infection either as pulpitis or localised dental abscess. Three quarters of the patients (both adult and child) had antibiotics prescribed for pulpitis. Pulpitis is inflammatory in nature and local treatment only could have removed and relieved the pain and infection. ${ }^{11}$ In fact the majority of the children were suffering from a local infection and the use of antibiotics as a treatment option was not entirely appropriate.

Seventeen adult patients had a cellulitic infection. This is usually indicated by the presence of a diffuse swelling, increased temperature, malaise and lymphadenitis and at the very least requires systemic antibiotics. However if the antibiotics are administered without drainage then the patient must be carefully monitored. ${ }^{12}$ This in itself would prove difficult for practitioners working on the emergency dental clinic rota.

The most common antibiotic prescribed was amoxicillin, either alone or for adults only, in combination with metronidazole. Both have been supported for their use in treating a dental abscess by microbiological and clinical findings. ${ }^{13-14}$ These antibiotics should however, only be used as an adjunct to the management of acute dentoalveolar abscess and not treatment.

Why the practitioners prescribed inappropriately is not known. However, some explanations can be put forward. Firstly, the practitioners may have a poor understanding of the pathological processes involved in pulp and periapical diseases. ${ }^{15}$ Furthermore, there could be a lack of knowledge of the indications for effective antibiotic use.

A second explanation is that attitudes to prescribing could be modified by the effects of the local environment. It is known that at the emergency clinics there is no appointment system and a large numbers of patients are seen in succession. This may restrict the dentist's ability to make a diagnosis, or limit the amount of time for surgical treatment that can be attempted. It is possible that facilities were not available in the emergency dental centres for the provision of surgical treatment. In a recent study by Palmer et al, ${ }^{7}$ lack of time and uncertainties of diagnosis were cited as reasons for antibiotic prescribing. Appropriate treatment decisions may therefore have been influenced by pressures within the system and antibiotics inappropriately prescribed to both adult and child patients as the first line of treatment.
What then is the way forward? Qualitative research is required to see if we can find out directly from practitioners why this problem of inappropriate antibiotic prescribing is so intractable. Methods also need to be developed to enable practitioners to change their prescribing behaviour. One such method is audit. Within medical practice antibiotic prescribing has been the subject of many audits. ${ }^{16,17}$ One of the few dental audits on antibiotic prescribing was reported in 1997 by Steed and colleagues. ${ }^{18}$ The audit looked at dental practitioners antibiotic prescribing regimes. Subsequently, a consensus was achieved on the rationale for antibiotic prescribing and the number of prescriptions issued fell by $50 \%$. In addition, since the completion of the study, the Faculty of General Dental Practitioners, Royal College of Surgeons have published guidelines for antibiotic prescribing. ${ }^{19}$ There is a need to investigate the effects of the guidelines upon practitioner's antibiotic prescribing regimes.

\section{Conclusion}

The majority of the patients attending the emergency dental clinics had pain (879/1011), associated with a localised infection either as a pulpitis (311/879) or a localised dental abscess (248/879) with only a minority (56/1011) showing signs/symptoms of a possible spreading infection. Three-quarters of patients (adult and child) with pulpitis were prescribed antibiotics with no surgical intervention. This study lends support to the hypothesis that antibiotics are being inappropriately prescribed by the dental profession.

1 General Dental Council (Maintaining Standards) November 1997: Providing for dental emergencies and out of hour's care, paragraph 3.11.

2 Olson A K, MacEdington E, Kulid J C, Weller R N. Update on antibiotics for endodontic practice. Compend Continuing Educ Dent 1995; 11:328-332.

3 Pogrel M A. Antibiotics in general practice. Dent Update 1994; 21: 274-270.

4 Mata E, Koren L Z, Morse D R, Sinai I H. Prophylatic use of penicillin V in teeth with necrotic pulps and asymptomatic periapical radiolucencies. Oral Surg Oral Med Oral Pathol 1985; 60: 201-207.

5 Thomas D W, Satterthwaite J, Absi E G, Lewis M A O, Shepard J P. Antibiotic prescription for acute dental conditions in the primary care setting. Br Dent J 1996; 181: 401-404.

6 Palmer N A O, Pealing R, Ireland R S, Martin M V. A study of prophylatic antibiotic prescribing in National Health Service general dental practice in England. Br Dent J 2000; 189: 43-46.

7 Palmer N A O, Pealing R, Ireland R S, Martin M V. A study of therapeutic antibiotic prescribing in National Health Service general dental practice in England. Br Dent J 2000; 188: 554-558.

8 Palmer N A O, Ireland R, Palmer S. Antibiotic prescribing patterns of a group of general dental practitioners: results of a pilot study. Prim Dent Care 1998; 4: 137-141.

9 Baker G R, Qualtrough A J E. An investigation into antibiotic prescribing at a dental teaching hospital. Br Dent J 1987; 162: 303-306.

10 SPSS for Windows Base Version 9.0.0. SPSS Inc, Chicago. 1998

11 Matthews R W, Peak J D, Scully C. The efficacy of management of acute dental pain. Br Dent J 1994; 176: 413-416.

12 Martin M V, Longman L P, Hill J B, Hardy P. Acute dentoalveolar infections: an investigation of the duration of antibiotic therapy. Br Dent J 1997; 183: 135-137.

13 Lewis M A O, McGowan D A, MacFarlane T W. Short- course high dosage Amoxicillin in the treatment of acute dentoalveolar abscess. Br Dent J 1986; 161: 299-302.

14 Fazakerley M W, McGowan P, Hardy P, Martin M V. A comparative study of cephadrine, amoxicillin and phenoxymethylpenicillin in the treatment of acute dentoalveolar infection. Br Dent J 1993; 174: 359-363.

15 Abbott P V. Selective and intelligent use of antibiotics in endodontics. Aust Endo J 2000; 26: 30-39.

16 De Santis G, Harvey K J, Howard D, Mashford M L, Moulds R F. Improving the quality of antibiotic prescription patterns in general practice. The role of educational intervention. Med J Aust 1994; 160: 502-505.

17 Swann R A, Clark J. Antibiotic policies - relevance to general practitioner prescribing. Family Health Services Authority, Great Britain. J Antimicrob Chemother 1994; 33: 131-135.

18 Steed M, Gibson J. An audit of antibiotic prescribing in general dental practice. Prim Dent Care 1997; 4: 66-70.

19 Faculty of General Dental Practitioners (UK) Royal College of Surgeons, England. Adult antimicrobial prescribing in primary dental care for general dental practitioners, 2000. 